\title{
PSYCHOLOGICAL CHARACTERISTICS OF PATIENTS WITH DIABETES
}

\author{
ANNAMÁRIA PÁPAI ${ }^{1}$, MARIA MELANIA COZMA², LUCICA EMILIA COȘA ${ }^{3}$, \\ ADRIANA MIHAI \\ 1,2, 3, 4 “George Emil Palade” University of Medicine, Pharmacy, Science, and Technology of Târgu Mureş
}

\begin{abstract}
Keywords: diabetes, Abstract: The aim of this study is to establish the psychological characteristics of patients with personality, $\quad$ coping diabetes. In the present study we included subjects with diabetes $(n=60)$, and control group $(n=62)$. In strategies, cognitive order to study the psychological characteristics of patients with diabetes, we have applied the schemes, irrational beliefs following tests: Zuckerman-Kuhlman Personality Questionnaire (ZKPQ), Strategic Approach to Coping Scale (SACS), Cognitive Emotion Regulation Questionnaire (CERQ), Young Schema Questionnaire (YSQ-S3), Attitudes and Beliefs Scale (ABS II). The results of this study outlined psychological characteristics of patients with diabetes which consists of neurotic, anxious personality with a high level of activity, dysfunctional coping strategies like: indirect action, antisocial action, aggressive action and catastrophizing, irrational thinking and few maladaptive cognitive patterns. The study highlighted the need to restructure the maladaptive cognitive schemes, irrational beliefs, dysfunctional coping strategies for people with diabetes with a psychological program in order to improve endocrine symptoms and increase quality of life.
\end{abstract}

Few studies $(1,2)$ have highlighted the importance of stress factors in determining, causing and maintaining endocrine diseases, but there is less information on how these events and stressors mediate endocrine diseases through psychological factors.

Regarding the psychological features of people with psychosomatic diseases in a study (3) conducted by evaluating people suffering from different types of psychosomatic diseases (hyperthyroidism, obesity, heart disease, diabetes etc.) it was highlighted that these people often feel guilty, they are tense, they need the approval of people around them, they are dependent and they are afraid to express their feelings of being rejected.

Adaptation to chronic diseases is defined as the degree of facing both physiologically and psychologically the stress caused by the condition of living with a chronic disease.(4) In case of diabetes, adaptation includes the personal involvement of the individual in drug treatment and ensuring the psychological adaptation to the disease, as they are interdependent and simultaneous.(5) Low psychological adjustment in patients with diabetes is associated with poor metabolic control, poor self-management (6) and lower quality of life.(7)

Patients with diabetes have psychological symptoms such as: negative emotional states in response to the chronic disease and its requirements, worry about complications, fear of hypoglycemia, feelings of guilt due to uncontrolled blood sugar and depression. $(8,9)$ People with diabetes suffer losses, narcissistic injuries, self-blame and guilt.(10)

A relationship between diabetes and emotional instability has also been detected.(11) In this study, it is shown that alexithymia (inability to distinguish between emotions, thoughts and physiological responses and difficulty in recognizing and describing emotions) is frequently associated with diabetes.(11) People with alexithymic traits are generally prone to develop physical illness (12) and in patients with diabetes, alexithymia correlate with higher levels of perceived stress and difficulty monitoring body signals, resulting in poor metabolic control.(13)

According to specialized studies $(14,15)$ there is a correlation between personality type D and diabetes. People with type 2 diabetes have a personality type $\mathrm{D}$, are introverted, emotionally unstable, and have negative emotions and social inhibition.(16) Type D personality is characterized by a mixture of high levels of negative affectivity and social inhibition, which leads to a stable tendency to experience negative emotions and inhibition of the expression of these emotions.(14) In a study (17) of patients with type 1 and type 2 diabetes, it was found that people with type 1 diabetes have significantly higher scores on the type A personality than people with type 2 diabetes. Type A personality is characterized by hostility, an internalized anger that generates excessive competitiveness with aggression, perfectionism (high expectations of oneself and others), exaggerated ambition.(18)

People with a high score of neuroticism are generally more prone to all types of somatic diseases.(19) The personality of patients with type 2 diabetes is characterized by neuroticism and psychoticism and shows a negative correlation with extraversion.(20)

Regarding patients with diabetes and their coping strategies, a study (21) suggests that active problem-focused coping behaviour and adequate social support promote adherence to diabetes regimens and may act as a buffer against complications and on quality of life in patients with type 1 diabetes. Frequent use of avoidant coping strategies by patients with diabetes have been associated with fewer blood glucose checks, less common self-care behaviours, and lower glycemic

${ }^{1}$ Corresponding author: Annamaria Papai, Str. Lalelelor, Tg. Mureş, România E-mail: annamariaporkolab@yahoo.com, Phone: +40745315332 Article received on 05.08.2020 and accepted for publication on 02.12.2020 
index at next assessment.(22)

According to a study (23) emotion-focused coping strategies appear to play a role in developing depressive symptoms, anxiety and maintaining stress related to type 2 diabetes. People with type 2 diabetes more often use emotion focused coping strategies than the people from the control group.(24) Restructuring maladaptive coping strategies through various psychological therapies applied to patients with diabetes may increase their resilience.(25)

Maladaptive cognitive patterns from childhood and unmet basic needs are correlated with a general propensity for psychosomatic illness.(26) Regarding diabetes and cognitive patterns, the results of a study (27) showed that patients with type 2 diabetes have significantly higher scores on some cognitive patterns such as: abandonment, failure, vulnerability, self-sacrifice than the control group.

Our study brings a new contribution in scientific field, approaching the psychosomatic perspective of diabetes, and highlights the relations between personality, coping strategies, irrational beliefs, cognitive schemes and diabetes.

\section{AIM}

The aim of this study is to establish the psychological characteristics (personality, coping strategies, irrational beliefs, cognitive schemes) of patients with diabetes compared to the control group, in order to create the most effective psychotherapeutic interventions for supporting drug treatments.

\section{MATERIALS AND METHODS}

The study has ethical approval from the George Emil Palade University of Medicine, Pharmacy, Science, and Technology of Târgu Mureş. The participants were informed about the study before participation and informed consent forms were obtained from all the subjects.

The study was done with two groups of subjects: diabetes $(n=60)$ and control $(n=62)$. The recruitment was from all patients that attended an endocrinology outpatient clinic between February 2020 - May 2020. Inclusion criteria were the following: patients diagnosed in last three years with diabetes according to a specialist. Exclusion criteria were the following: refuse to study participation, patients without a specialist diagnosis, more than 3 years from onset of the disease, already included in psychotherapy, incapacity to write or to understand the test demands, incomplete data collection. (Total number of patients excluded 28.) The control group consisted of 62 healthy volunteers.

The applied psychological instruments were the following five questionnaires. The questionnaires were validated on the Romanian population. For the personality assessment we applied the Zuckerman-Kuhlman Personality Questionnaire Form III Revised (ZKPQ). Zuckerman-Kuhlman Personality Questionnaire Form III Revised (ZKPQ) (28) was developed to evaluate five features. The questionnaire measures five personality dimensions: impulsive thrill seeking, neuroticismanxiety, aggression-hostility, sociability, activity, social desirability.

For evaluating coping strategies, we applied the Strategic Approach to Coping Scale (SACS) and Cognitive Emotion Regulation Questionnaire (CERQ)

The Strategic Approach to Coping Scale (SACS) measures behavioural coping strategies in a social context, which are used after experiencing certain negative events or situations. The SACS scale includes nine subscales: assertive action, social relations, seeking social support, prudent course of action, instinctive action, avoidance, indirect action, antisocial action, aggressive action.(29)

The Cognitive Emotion Regulation Questionnaire
(CERQ) is identifying adaptive or maladaptive cognitive coping strategies when people facing negative events.(30) CERQ questionnaire measures the frequency of use of certain strategies through 9 rating scales: self-blame, acceptance, rumination, positive refocusing, refocus on planning, positive reappraisal, putting into perspective, catastrophizing, blaming others

For assessing cognitive schemes, we used the Young Schema Questionnaire (YSQ-S3) (31), 114 items are divided into the following 18 subscales: emotional deprivation (ED), abandonment/instability (AB), mistrust/abuse (MA), social isolation/alienation (SI), defectiveness/shame (DS), failure (FA), dependence/incompetence (DI), vulnerability to harm or illness (VH) enmeshment/undeveloped self (EM), entitlement/grandiosity (ET), insufficient self-control/selfdiscipline (IS), subjugation (SB), self-sacrifice (SS), approvalseeking/recognition-seeking (AS), negativity/pessimism (NP), emotional inhibition (EI), unrealistic standards/hypercriticism (US), punitiveness (PU).

For assessing a person's attitudes and beliefs we used Attitudes and Beliefs Scale (ABS II). Attitudes and Beliefs Scale (ABS II) evaluates the irrational and rational beliefs described in Albert Ellis's theory.(32) The scale contains 72 items. The efficiency of the tool is enhanced by the fact that it allows to calculate separate scores on different types of irrational beliefs: IB (irrational beliefs), RB (rational beliefs) the imperative "must" (DEM), global depreciation and evaluation (SD / GE), low tolerance to frustration (LFT), catastrophic thinking (AWF), as well as the estimation of some global values $\mathrm{T}$ (total) rationality / irrationality.

These were pen-a-paper tests. Total duration of psychological evaluation was 120 minutes.

The statistical analyzes were conducted using the SPSS 22.00 package software: descriptive analysis: mean, standard deviation, inferential statistics: Independent Sample T Test, Pearson correlation. The results were evaluated at the $\mathrm{p}<0,05$ significance level.

\section{RESULTS}

In the present study we evaluated the difference between patients with diabetes and a control group regarding personality, cognitive schemes, rational/irrational beliefs and coping strategies. Subjects with diabetes $(n=60)$ were between 37 and 69 years old, the average age was 51,28 the standard deviation is 8,410 . Regarding the environment of origin and gender, 21 patients were from rural areas, 39 from urban areas, 44 women and 16 men.

The control group consisted of 62 healthy volunteers of which 57 women and 5 men, aged between 19 and 66 years, the average age was 39.58 , the standard deviation 10.078 . Regarding the environment of origin, 21 were from rural areas and 41 from urban areas.

Analyzing the relationship between personality and diabetes, the results show significant differences between patients with diabetes and the control group (Table 1) in terms of neuroticism / anxiety $(\mathrm{t}(96,157)=2,435, \mathrm{p}=, 017)$ and activity $(\mathrm{t}(120)=2,129, \mathrm{p}=, 035)$. Neuroticism / anxiety and activity being higher in patients with diabetes.

Neuroticism correlates positively with aggression / hostility $(, 501 * *)$ and negatively with sociability $\left(-, 287^{*}\right)$.

Regarding constructive and adaptive coping strategies, significant differences between patients with diabetes and the control group (table no. 1.) highlighted the acceptance ( $\mathrm{t}(120)=$ $2,185, \mathrm{p}=, 031)$, positive refocusing $(\mathrm{t}(120)=2,017, \mathrm{p}=, 046)$, putting into perspective $(\mathrm{t}(112,492)=3,282, \mathrm{p}=, 001)$, and prudent course of action $(t(120)=3,155, p=, 002)$. Each of these coping strategies are used more often by patients with diabetes than the control group. 
PUBLIC HEALTH AND MANAGEMENT

Table no. 1. Statistically significant difference on the psychological factors assessed by comparing patients with diabetes and the control group

\begin{tabular}{|c|c|c|c|c|c|c|}
\hline Psychological factors evaluated & $\begin{array}{l}\text { Mean/SD } \\
\text { Diabetes }\end{array}$ & $\begin{array}{c}\text { Mean/SD } \\
\text { Control group } \\
\end{array}$ & $\mathbf{t}$ & df. & $\begin{array}{l}\text { Sig. } \\
\text { p }\end{array}$ & d \\
\hline \multicolumn{7}{|l|}{ Personality (ZKPQ) } \\
\hline Neuroticism/anxiety & $5,08 \pm 4,511$ & $3,44 \pm 2,714$ & 2,435 & 96,157 &, 017 & 0,50 \\
\hline Activity & $9,50 \pm 3,265$ & $8,31 \pm 2,923$ & 2,129 & 120 &, 035 & 0,39 \\
\hline \multicolumn{7}{|l|}{ Coping strategies (SACS) } \\
\hline Prudent course of action & $19,20 \pm 2,916$ & $17,39 \pm 3,404$ & 3,155 & 120 & ,002 & 0,58 \\
\hline Indirect action & $10,92 \pm 4,183$ & $8,11 \pm 3,255$ & 4,122 & 111,396 &, 000 & 0,78 \\
\hline Antisocial action & $11,30 \pm 4,196$ & $9,82 \pm 3,457$ & 2,126 & 120 &, 036 & 0,39 \\
\hline Aggressive action & $12.53 \pm 2,843$ & $11.34 . \pm 2,891$ & 2,300 & 120 & 023 & 0,42 \\
\hline \multicolumn{7}{|l|}{ (CERQ) } \\
\hline Acceptance & $13,15 \pm 3,156$ & $11,95 \pm 2,900$ & 2,185 & 120 &, 031 & 0,40 \\
\hline Positive refocusing & $13,87 \pm 3,993$ & $12,48 \pm 3,575$ & 2,017 & 120 &, 046 & 0,37 \\
\hline Putting into perspective & $13,57 \pm 3,693$ & $11,58 \pm 2,934$ & 3,282 & 112,492 & 001 & 0,62 \\
\hline Catastrophizing & $7,02 \pm 2,813$ & $6,19 \pm 1,638$ & 1,967 & 94,251 &, 052 & 0,41 \\
\hline \multicolumn{7}{|l|}{$\begin{array}{l}\text { Attitudes and Beliefs } \\
\text { (ABS-II) }\end{array}$} \\
\hline Totally rational / irrational & $95,83 \pm 37,595$ & $76,08 \pm 31,649$ & 3,143 & 120 & ,002 & 0,57 \\
\hline Irrationality & $56,05 \pm 23,695$ & $47,37 \pm 20,538$ & 2,172 & 120 &, 032 & 0,40 \\
\hline DEM - The imperative must & $25,75 \pm 10,441$ & $21,56 \pm 9,683$ & 2,297 & 120 & 023 & 0,42 \\
\hline $\begin{array}{l}\mathrm{SD} / \mathrm{GE}-\text { Depreciation and global } \\
\text { valuation }\end{array}$ & $18,92 \pm 10,723$ & $13,37 . \pm 6,727$ & 3,409 & 98,656 & ,001 & 0,69 \\
\hline LFT - Low tolerance to frustration & $25,80 \pm 9,880$ & $22,11 . \pm 9,744$ & 2,075 & 120 & 040 & 0,38 \\
\hline AWF -Catastrophic thinking & $25,36 \pm 9,136$ & $20.73 . \pm 9,468$ & 2,847 & 120 & 005 & 0,52 \\
\hline \multicolumn{7}{|l|}{ Cognitive scheme (YSQ-S3) } \\
\hline Deficiency/Shame & $9,38 \pm 4,211$ & $8,34 \pm 3,224$ & 2,333 & 89,852 &, 022 & 0,49 \\
\hline Emotional Inhibition & $13,18 \pm 4,608$ & $11,40 \pm 4,507$ & 2.157 & 120 &, 033 & 0,39 \\
\hline
\end{tabular}

Regarding maladaptive coping strategies, significant differences between patients with diabetes and the control group (table no. 1.) highlighted the indirect action $(\mathrm{t}(111,396)=4,122$, $\mathrm{p}=, 001)$, antisocial action $(\mathrm{t}(120)=2,126, \mathrm{p}=, 036)$, aggressive action $(\mathrm{t}(120)=2,300, \quad \mathrm{p}=, 023) \quad$ and catastrophizing $(\mathrm{t}(146,882)=3,874, \mathrm{p}=, 052)$. Each of these coping strategies are used more often by patients with diabetes than the control group.

The Attitudes and Beliefs Scale (ABS II) showed a statistically significant difference between patients with diabetes and the control group in the overall assessment of irrational / rational beliefs and attitudes $(\mathrm{t}(120)=3,143, \mathrm{p}=, 002)$, these being higher in patients with diabetes. Significantly higher scores were obtained by patients with diabetes compared to the control group at the following subscales/factors of the Attitudes and Beliefs Scale (ABS II): irrationality $(\mathrm{t}(120)=2,172, \mathrm{p}=, 032)$, the imperative "must" ( $\mathrm{t}(120)=2,297, \mathrm{p}=, 023)$, depreciation and global evaluation ( $(98,656)=3,409, \mathrm{p}=, 001)$, low tolerance to frustration $(\mathrm{t}(120)=2,075, \mathrm{p}=, 040)$ and catastrophic thinking $(\mathrm{t}(120)=2,847, \mathrm{p}=, 008)$.

Statistically significant differences between patients with diabetes and the control group exist in the following cognitive schemes: deficiency/shame $(\mathrm{t}(89,852)=2,333$, $\mathrm{p}=, 022)$, emotional inhibition $(\mathrm{t}(120)=2.157, \mathrm{p}=, 033)$, According to standard means and deviations (Table 1) all these cognitive schemes are used more frequently by patients with diabetes than the control group.

Neuroticism (as a personality trait) in patients with diabetes correlates positively with the following maladaptive cognitive patterns: abandonment/instability $\left(, 379^{* *}\right)$, mistrust/abuse $\left(, 260^{*}\right)$, social isolation/alienation $\left(, 264^{*}\right)$, defectiveness /shame $\left(, 318^{*}\right)$, failure $\left(, 345^{* *}\right)$, vulnerability to harm or illness $(, 278 *)$ negativity/pessimism $(, 261 *)$, emotional inhibition $\left(, 278^{*}\right)$, unrealistic standards hypercriticism $\left(, 347^{* *}\right)$, punitiveness $\left(, 377^{* *}\right)$.

\section{DISCUSSIONS}

Studies in the field $(14,17)$ describe differently the personality of patients with type 1 and type 2 diabetes. The personality of patients with type 2 diabetes is characterized by neuroticism and psychoticism and shows a negative correlation with extraversion (33), and diabetes type 1 is characterized by internalized anger that generates excessive competitiveness colored with aggression, perfectionism.(17)

Following the processing of the results, the patients with diabetes, in terms of personality, presented significantly higher results from a statistical point of view to neuroticism / anxiety and activity than the control group. The high level of anxiety and neuroticism were also highlighted in other studies $(8,9,33)$ but the need for high activity of patients with diabetes was outlined only in the present study. Neuroticism as a personality trait in patients with diabetes correlates positively with aggression (also as a personality trait) and negatively correlates with sociability.

Life events are not stressful in themselves, their level of stress depends on how a person interprets the event. Depending on the interpretation, people may react differently to stressful events. These cognitive, emotional and behavioural reactions are coping strategies. People in stressful situations can react adaptively by accepting and resolving the situation or maladaptively by attack / aggression: verbal or physical or by avoidance.(34) These dysfunctional reactions can cause various psychosomatic and chronic diseases. Several studies $(35,36)$ have shown that maladaptive coping strategies are characteristic of people with diabetes. The use of maladaptive coping strategies (e.g., avoidance) predicts several quality-of-life problems and depressive symptoms in patients with type 1 diabetes.(37) Another study (38) conducted with adolescents with type 1 diabetes reported that they use coping strategies such as: adaptation, acceptance and avoidance.

In the present study, both adaptive and maladaptive coping strategies were highlighted in patients with diabetes. They use adaptive coping strategies such as: acceptance, positive refocusing, putting in to perspective, and prudent course of action. Acceptance is an adaptive coping strategy but if used without taking action to resolve the stressful situation it can become a dysfunctional coping strategy and can cause emotional 


\section{PUBLIC HEALTH AND MANAGEMENT}

problems: anxiety and depression.(30) The maladaptive coping strategies used by patients with diabetes are: indirect action, antisocial action, aggressive action and catastrophizing.

There are not many studies regarding the beliefs of patients with diabetes. In our study, patients with diabetes presented statistically significantly higher scores on all factors of the questionnaire of attitudes and beliefs towards the control group. Patients with diabetes have irrational thinking with high scores on subfactors: the imperative "must", depreciation and global evaluation, low tolerance to frustration and catastrophic thinking.

A study (39) done with patients with diabetes revealed a high level of maladaptive cognitive patterns in people with diabetes, while following cognitive behavioral therapy these cognitive illnesses were restructured into adaptive cognitive patterns. In the present study, two cognitive patterns were highlighted in patients with diabetes: deficiency / shame, emotional inhibition. Deficiency / shame refers to the fact that patients with diabetes feel worthless, incapable in certain important aspects of life and they think that if others notice this, they won't appreciate them, at the same time they are very sensitive to criticism. Inhibition of emotions and feelings patients with diabetes use it to avoid disapproval of others and not to lose control over their own impulses. This is somewhat consistent with the results of the study (11) which showed that patients with diabetes have alexithymia (inability to distinguish between emotions, thoughts and physiological responses and difficulty in recognizing and describing emotions).

\footnotetext{
CONCLUSIONS

The results of this study have outlined psychological characteristics of patients with diabetes. These psychological characteristics of people with diabetes contain neurotic, anxious personality with a high level of activity, dysfunctional coping strategies, irrational thinking and few maladaptive cognitive patterns. By knowing personality specifics and ways to cope with stress of patients with diabetes, we will be able to create and build specific psychological therapies for patients with diabetes. In the future, based on these results, we would like to develop and implement an intervention program based on cognitive behavioural therapies, for people with diabetes, through which we will restructure these psychological characteristics of people with diabetes leading to maladaptive behaviours and thus maintain or determine the state of physical illness.
}

\section{REFERENCES}

1. Chrousos GP. Stress and disorders of the stress system. Nature reviews endocrinology. 2009;5:7:374

2. Boscarino JA. Posttraumatic stress disorder and physical illness: results from clinical and epidemiologic studies. Annals of the New York Academy of Sciences. 2004;1032:1:141-153.

3. Trifu S. Mental Fragility And Psychosomatic Illness Romanian Journal of Psychiatry. 2011;13:1:37-44.

4. Whittemore R, Jaser S, Guo J, Grey M. A conceptual model of childhood adaptation to type 1 diabetes. Nursing outlook. 2010;58:5:242-251.

5. Ebrahimi H, Moonaghi HK, Jafarabadi MA, et al. Development and Preliminary Validation of Diabetes Adjustment Assessment Scale (DAAS): a New Measure of Adjustment with Type 2 Diabetes. Journal of caring sciences. 2016;5:2:145-152.

6. Schmitt A, Reimer A, Kulzer B, et al. Assessment of diabetes acceptance can help identify patients with ineffective diabetes self-care and poor diabetes control. Diabetic Medicine. 2014;31:11:1446-1451.
7. Misra R, Lager J. Predictors of quality of life among adults with type 2 diabetes mellitus. Journal of Diabetes and its Complications. 2008;22:3:217-223.

8. Dennick K, Sturt J, Speight J. What is diabetes distress and how can we measure it? A narrative review and conceptual model. Journal of Diabetes and its Complications. 2017;31:5:898-911.

9. Wardian JL, Tate J, Folaron I, et al. Who's distressed? A comparison of diabetes-related distress by type of diabetes and medication. Patient education and counseling. 2018;101:8:1490- 1495 .

10. Marchini F, Caputo A, Napoli A, et al. Chronic illness as loss of good self: underlying mechanisms affecting diabetes adaptation. Mediterranean Journal of Clinical Psychology. 2018;6:3

11. Gentili P, Gentile L. Diabete e alessitimia: Stato attuale e prospettive [Diabetes and alexithymia: Current status and perspectives]. Giornale Italiano di Diabetologia e Metabolismo. 2010;30:65-70

12. Di Trani M, Mariani R., Renzi A, et al. Alexithymia according to Bucci's multiple code theory: A preliminary investigation with healthy and hypertensive individuals. Psychology and Psychotherapy: Theory, Research and Practice. 2018;91:2:232-247.

13. Avci D, Kelleci M. Alexithymia in patients with type 2 diabetes mellitus: The role of anxiety, depression, and glycemic control. Patient preference and adherence. 2016;10:1271-1277.

14. Bechter K, Martin Wieland M, Hamman GF Chronic Cervicogenic Tinnitus Rapidly Resolved by Intermittent Use of Cervical CollarFront. Psychiatry. 2016;23:3.

15. Conti C, Di Francesco G, Fontanella L, et al. Negative affectivity predicts lower quality of life and metabolic control in type 2 diabetes patients: a structural equation modeling approach. Frontiers in Psychology. 2017;8:831.

16. van Dooren FE, Denollet J, Verhey F R, et al. Psychological and personality factors in type 2 diabetes mellitus, presenting the rationale and exploratory results from The Maastricht Study, a population-based cohort study. BMC psychiatry. 2016;16:1:17.

17. Rouland A, Chauvet-Gelinier JC, Sberna AL, et al Personality types in individuals with type 1 and type 2 diabetes. Endocrine Connections. 2020;9:3:254-260.

18. Chauvet-Gélinier JC, Trojak B, Lemogne C, et al. Potential influence of Type A personality on plasma C-reactive protein levels in people with diabetes. Diabetes and Metabolism. 2016;42:88-95.

19. Deary IJ, Weiss A, Batty GD. Intelligence and personality as predictors of illness and death: How researchers in differential psychology and chronic disease epidemiology are collaborating to understand and address health inequalities. Psychological science in the public interest. 2010;11:2:53-79

20. Kontoangelos K, Papageorgiou CC, Raptis AE, et al. Cytokines, diabetes mellitus and psychopathology: a challenging combination. Neuroendocrinology Letters. 2014;35:2.

21. Wasserman LI. Diabetes mellitus as a model of psychosomatic and somatopsychic interrelationships. The Spanish journal of psychology. 2006;9:1:75-85.

22. Iturralde E, Weissberg-Benchell J, Hood KK. Avoidant coping and diabetes-related distress: pathways to adolescents' type 1 diabetes outcomes. Health Psychology. 2017:36:3:236.

23. Burns RJ, Deschênes SS, Schmitz N. Associations between coping strategies and mental health in individuals with type 2 diabetes: Prospective analyses. Health Psychology. 
2016;35:1:78.

24. Aktar R, Satu FY. Perceived stress, coping strategies and psychological well-being of people with Diabetes and people without Diabetes in Bangladesh: A comparative study. Jurnal Psikologi Malaysia. 2017;31:1.

25. Yi-Frazier JP, Yaptangco M, Semana S, et al. The association of personal resilience with stress, coping, and diabetes outcomes in adolescents with type 1 diabetes: Variable-and person-focused approaches. Journal of health psychology. 2015;20(9):1196-1206.

26. Panic D, Stanojevic TS, Pesic MH. Early maladaptive schemas among psychosomatic prone individuals. Ceskoslovenska psychologie. 2016;60:1:1.

27. Mirdrikvand F, Sepahvandi MA, Khodarahimi S, et al. Early Maladjustment Schemas in Individuals with and without Type 2 Diabetes Mellitus. Journal of Mind and Medical Sciences. 2019;6:1:150-156.

28. Opre A, Albu M. Psychometric properties of the Romanian version of the Zuckermen-Kuhlman Personality Questionnaire (ZKPQ): preliminary data. An. Inst. de Ist. „G. Bariţiu”. Cluj-Napoca: Series Humanistica. 2010;(8):85-100.

29. Budau O, Albu M. SACS: Strategic Approach to Coping Scale. Cluj-Napoca: ASCR Publishing; 2010. p. 25-33.

30. Perțe A, Țincaș I. Handbook for use of the Cognitive Emotion Regulation Questionnaire - Adaptation and standardization of CERQ on the population in Romania. Cluj Napoca: ASCR Publishing; 2009. p. 468-502.

31. David D. Young cognitive schema questionnaire, short form (YSQ - S3) manual CASS. Cluj-Napoca: ASCR Publishing; 2010. p. 8-9.

32. David D. ABS II scale of attitudes and beliefs, CASS manual + Romanian version. Cluj-Napoca: ASCR Publishing; 2010. p. 4-7.

33. Kontoangelos K, Papageorgiou CC, Raptis AE, et al. Cytokines, diabetes mellitus and psychopathology: a challenging combination. Neuroendocrinology Letters. 2014;35:2.

34. Pápai A, Cozma M, Coșa LE, Mihai A. Coping strategies in thyroid desease Acta Medica Transilvanica. 2020;25(2):2932.

35. Jaser SS, White LE. Coping and resilience in adolescents with type 1 diabetes. Child Care Health Dev. 2011;37(3):335-42.

36. Yi-Frazier JP, Smith RE, Vitaliano PP, et al. A PersonFocused Analysis of Resilience Resources and Coping in Diabetes Patients. Stress Health. 2010;26(1):51-60.

37. Jaser SS, Patel N, Xu M, et al. Stress and coping predicts adjustment and glycemic control in adolescents with type 1 diabetes. Annals of behavioural medicine. 2017;51(1):3038 .

38. Hapunda G, Abubakar A, Van de Vijver F, Pouwer F. Living with type 1 diabetes is challenging for Zambian adolescents: qualitative data on stress, coping with stress and quality of care and life. BMC endocrine disorders. 2015;15(1):20.

39. Oieru DS, Popa AR, Vlad I. The impact of psychological interventions on the quality of life of diabetes mellitus patient. Romanian Journal of Diabetes Nutrition and Metabolic Diseases. 2014;21(4):301-311. 